\title{
Oral Radiology accepted by Medline
}

\author{
Junichi Asaumi ${ }^{1}$
}

Published online: 22 December 2018

(c) Japanese Society for Oral and Maxillofacial Radiology and Springer Nature Singapore Pte Ltd. 2018

Dear Readers,

I am very proud to announce that $\operatorname{Oral}$ Radiology $(O R)$ has been accepted by Medline.

That result is thanks to numerous efforts by the first editor of $O R$, Hajime Fuchihata, Osaka University, as well as to those of subsequent editors, Kanji Kishi, Okayama University and Keiji Tanimoto, Hiroshima University, for a period of over 30 years.

I am also deeply grateful for the work of the associate editors of OR: Allan G Farman, University of Louisville School of Dentistry; Takafumi Hayashi, Niigata University; Reinhilde Jacobs, KU Leuven; Yasuhiro Morimoto, Kyushu Dental College; Kaan Orhan, Ankara University; Jie Yang, Temple University; and David MacDonald-Jankowski, The University of British Columbia.

In addition, I would like to express my thanks to members of the International Editorial Board: Jinwu Chen, Fourth Military Medical School; Yuk-Kwan Chen, Kaohsiung Medical University; Soon-Chul Choi, Seoul National University; Wichitsak Cholitgul, Chulalongkorn University; Malcolm I Coombs, Sydney Dental Hospital; Tazuko K Goto, Tokyo Dental College; Naoya Kakimoto, Hiroshima University; Sunali Khanna, Nair Hospital Dental College; Eun-Kyung Kim, Dankook University, College of Dentistry; Saulo Leonardo Sousa Melo, University of Iowa; Gerard CH Sanderink, Academic Center for Dentistry Amsterdam; Dania Tamimi, Orlando; Hiroshi Watanabe, Tokyo Medical and Dental University; Ann Wenzel, Aarhus University; and Yoshinobu Yanagi, Okayama University Hospital.
I would like to appreciate Assistant Editors, Miki Hisatomi, Okayama University and Rei Tanaka, University of Hong Kong for their help.

I appreciate financial support from the Japanese Society for Oral and Maxillofacial Radiology, the cooperation of international publisher Springer Nature, editing by Edanz Group Japan, Inc., and, above all, the support of $O R$ 's contributors, reviewers and readers.

I am delighted that I am able to send all of you this very happy news today. The recent application for $O R$ to be accepted by Medline was the fourth attempt since 2005, and I am so pleased at this outcome. I appreciate the understanding and cooperation you have shown in the past. In addition, I hope that I can look forward to your continuous support in the future. I also hope that as a truly international high-quality publication, our journal will further develop and publish breakthroughs in its field. I am eager for $O R$ to become a worthy source of pride in our society. Thank you very much again.

Junichi Asaumi, Editor-in-Chief, $O R$ Springer Nature

Publisher's Note Springer Nature remains neutral with regard to jurisdictional claims in published maps and institutional affiliations.
Junichi Asaumi

asaumi@md.okayama-u.ac.jp

1 Okayama University, Okayama, Japan 